\title{
Balkanologie
}

Balkanologie Revue d'études pluridisciplinaires

Vol. II, nº 1 | 1998

Volume II Numéro 1

\section{Le Parlement de Serbie adopte une nouvelle loi sur l'université}

\section{Slobodanka Ast}

Traducteur : Patrick Michels et Yves Tomić

\section{OpenEdition \\ Journals}

Édition électronique

URL : http://journals.openedition.org/balkanologie/253

DOI : 10.4000/balkanologie.253

ISSN : 1965-0582

\section{Éditeur}

Association française d'études sur les Balkans (Afebalk)

\section{Édition imprimée}

Date de publication : 1 juillet 1998

ISSN : $1279-7952$

\section{Référence électronique}

Slobodanka Ast, « Le Parlement de Serbie adopte une nouvelle loi sur l'université », Balkanologie [En ligne], Vol. II, n 1 | 1998, mis en ligne le 02 juin 2008, consulté le 17 décembre 2020. URL : http:// journals.openedition.org/balkanologie/253; DOI : https://doi.org/10.4000/balkanologie.253

Ce document a été généré automatiquement le 17 décembre 2020.

(C) Tous droits réservés 


\title{
Le Parlement de Serbie adopte une nouvelle loi sur l'université
}

\author{
Slobodanka Ast
}

Traduction : Patrick Michels et Yves Tomić

\section{NOTE DE L'ÉDITEUR}

Traduit du serbo-croate par Patrick Michels et Yves Tomić.

1 À l'aube du troisième millénaire, au Parlement de Serbie, les députés de la coalition rouge-brune (le parti socialiste de Milošević, la gauche unifiée yougoslave [JUL Jugoslovenska Udružena Levica], dirigée par la femme de ce dernier, Mirjana Marković, et les radicaux de l'extrême-droite du dr. Vojislav Šešelj) ont, unanimement et dans la bonne humeur, élaboré la loi la plus restrictive des 160 années d'existence de l'université de Belgrade.

2 Cette nouvelle loi supprime l'autonomie universitaire: les facultés sont désormais dirigées comme des entreprises. Le gouvernement nomme les recteurs, les doyens, et même les professeurs. Même à l'époque de la dictature du roi Alexandre, en 1929, ainsi qu'à l'époque de l'occupation allemande, lors de la Seconde Guerre mondiale, les recteurs et les doyens n'étaient pas désignés par les instances du pouvoir, mais par le conseil des enseignants.

3 La proposition de l'université de Belgrade, visant à reporter l'adoption de la loi à l'automne, et, qu'entre-temps, le gouvernement, en collaboration avec l'université, élabore une nouvelle version de la loi, n'a pas été acceptée. L'université de Belgrade n'a jamais connu une situation plus difficile, jamais auparavant, elle n'a eu aussi peu d'autonomie, ni sous les Obrenović, ni sous les Karađorđević, ni sous le Maréchal Tito ; telle est l'analyse du dr. Nebojša Popov, un des meilleurs connaisseurs de l'histoire contemporaine de l'université de Belgrade et auteur de nombreux ouvrages sur les relations entre le pouvoir et l'université. 
4 L'adoption de la nouvelle loi sur l'université a été suivie de manifestations d'étudiants et de professeurs de l'université de Belgrade : des cordons de la police spéciale ont été dressés autour du Parlement serbe et, comme lors des manifestations estudiantines de 1996-1997, les policiers ont battu et blessé gravement des étudiants, des professeurs, ainsi que des civils.

\section{Vengeance de la manifestation}

5 La campagne contre l'université a été planifiée de longue date : le comité universitaire de la JUL avait ouvertement fait savoir que la "gauche" réglerait ses comptes avec la droite. La nouvelle loi sur l'université représente, selon de nombreux analystes politiques de la situation en Serbie, les représailles à l'encontre des manifestations estudiantines qui se sont déroulées de novembre 1996 à mars 1997. Alors, les universitaires, aussi bien que les citoyens, ont franchement déclaré ce qu'ils pensaient tant du vol électoral que du régime de S. Milošević.

6 Le parlement serbe n'a accepté aucun des 66 amendements proposés par l'opposition. Le dr. Dragan Veselinov, député de la coalition de Voïvodine et professeur à l'université de Belgrade, déclare que cette loi, qui représente le symbole de l'intervention politique de l'État, devrait aboutir à la politisation de l'université, la plaçant sous contrôle étatique direct (les autorités, les cadres et les décisions universitaires devant répondre à l'État). D. Veselinov estime que cette loi deviendra un dangereux instrument de suppression des critiques scientifiques des processus sociaux du pays. Les représentants d'autres partis d'opposition estiment que les conséquences de cette nouvelle loi seront tragiques. Elle introduit de nouveau la notion oubliée de conformité morale et politique [de la période communiste] à l'université.

7 La loi sur l'université a été promulguée en secret, sans débat public, sans la participation d'universitaires, en plein milieu des examens de juin, mais aussi lors des dramatiques événements au Kosovo. Des commentaires cyniques ont fusé après l'adoption de la loi: le député de Voïvodine, Nenad Čanak, a déclaré que le gouvernement désignera bientôt même le président de l'Académie des sciences, comme c'était le cas avec Elena Ceauşesku, puis les époux Milošević attendront une fin similaire à celle de Nikolae et Elena.

8 N. Popov observe que le régime en Serbie, par la promulgation d'une telle loi, montre des signes de panique (...). Ce dernier affronte une plus grande panique que les citoyens, que les universitaires mêmes. Selon lui, les individus ont commencé à se libérer de la peur. Pourtant, à cause l'agressivité accrue du régime, du fait qu'il a, finalement, appauvri une grande partie de la population, ainsi que les universitaires qui ne vivent que de leur salaire (en moyenne 200 \$ par mois !), il serait dangereux que ce mouvement s'inverse et que la peur revienne (...). Si cela devait arriver, le pouvoir se renforcerait pour, de nouveau, produire du chaos, instaurer un nouvel ordre et empêcher ce qui serait normal pour tout pays normal : une alternative électorale.

9 Beaucoup dépend de l'université. Le régime belgradois le sait et c'est pour cela qu'il détruit ce qui permettrait au peuple de comprendre ce qui s'est déroulé, ce qui l'attend et ce qui pourrait changer, soutient N. Popov. 


\section{Vérités et mensonges}

10 Afin d'instaurer un contrôle de type soviétique sur l'université, les promoteurs de la nouvelle loi affirment qu'elle a été élaborée sur le modèle suédois ou français, qu'elle est en harmonie avec les lois modernes sur l'université.

Si de telles assertions peuvent s'entendre de la part du commun des mortels, les experts prouvent que le législateurs ment insolemment : il n'est pas vrai que dans un seul des pays développés, de la France aux États-Unis, les recteurs, les doyens, les professeurs, ... soient choisis en fonction de critères politiques.

12 La loi a été promulguée, entre autres, pour chasser la politique avec l'université, d'après le ministre de la culture, Jovo Todorović. Il a seulement réitéré l'idée du dr. M. Marković, présidente de JUL, qui soutenait que le projet de réforme et de reconstruction de la société yougoslave implique que les écoles, les universités, les institutions culturelles, mais également sanitaires, doivent être préservées des activités des partis politiques. L'ironie est qu'aucun parti n'a de comité universitaire, ni son club de députés, sauf la JUL et le SPS (Socijalistička Partija Srbije). Les membres de partis à l'université accusent ceux qui ne sont membres d'aucun parti de se mêler de politique.

Des professeurs de Belgrade considèrent que l'adoption de la loi est une ouverture pour une variante de la révolution culturelle chinoise dans les universités de Serbie, dont M. Marković, principale idéologue de la JUL, fait l'apologie depuis longtemps.

Peut-être que l'adoption de la loi sur l'université, votée en secret et avec facilité, n'ayant rencontré qu'une faible résistance universitaire et de l'opinion publique, représentera une victoire du régime qui, depuis des années, laisse derrière lui des "terres désolées" - de Bosnie et de Krajina au Kosovo, de l'économie à la formation. Autrefois, les professeurs idéologiquement "inaptes" et désobéissants étaient chassés de l'université. Aujourd'hui, la peur réapparaît ; une partie des universitaires humiliés, apprauvis et menacés dans leur existence ont peur des grandes ruptures et des purges dont l'État les menace. Toutefois, des protestations organisées et isolées apparaissent qui tentent de sauvegarder et de défendre l'autonomie de l'université des pressions de la coalition rouge-brune corrompue et inculte.

En vérité, on n'en est pas arrivé à la grève générale que souhaitaient les étudiants et le corps enseignant de la Faculté de philosophie à Belgrade, les autres centres universitaires de Serbie n'ayant pas réagi à l'appel. Pourtant, il ne faut pas négliger que la grande partie des facultés et instituts belgradois se sont prononcés, dans leurs conseils d'enseignement scientifique, contre cette loi restrictive; les doyens et vicesdoyens de six facultés ont présenté leur démission; ils ont expliqué les raisons de leur départ: "l'adoption de la nouvelle loi sur l'université représente une tentative de dégrader l'âme et l'essence de l'université de Belgrade. Les enseignants, les collaborateurs scientifiques et les étudiants de cette faculté ne doutent pas de leur détermination, avec tous les moyens légitimes de la lutte, à poursuivre la résistance à cette attaque contre la dignité de l'université ", déclare le professeur Fedor Zdanski, doyen démissionnaire de la Faculté de technologie-metallurgie de Belgrade. Srđan Hofman, recteur de la Faculté de musicologie, ajoute : «je refuse de prendre part aux funérailles de l'université. La loi est tout simplement fatale à l'instruction publique ». Le dr. Srbijanka Turajlić, professeur à la Faculté d'électrotechnique, affirme que la loi, 
entre autres, est contraire à la Charte des universités européennes que même la Serbie a signé, et que les professeurs continuent le combat contre cette nouvelle loi.

\section{Nous n'acceptons pas}

Plusieurs centaines de professeurs de l'université de Belgrade ont, dans une lettre ouverte, fait savoir que la nouvelle loi sur l'université est contraire à la civilisation, de par ses fondements conceptuels impliquant que toute la vie et le travail universitaires doivent être subordonnés au diktat de l'État :

de l'Académie de Platon et du Lycée d'Aristote aux universités modernes du XIX ème et $\mathrm{XX}^{\text {ème }}$ siècle, la liberté des débats et les courageuses recherches de la vérité représentent le fondement de chaque acquisition du savoir et sa transmission aux autres. L'université n'est pas, au contraire de ce que pensent les concepteurs de la nouvelle loi, une caserne, ni une usine, encore moins une entreprise d'État. Elle ne peut réaliser pleinement ses fonctions qu'en tant qu'association libre de professeurs et d'étudiants, menée par des personnalités choisies en fonction de leurs qualités professionnelles et morales, et non par des individus placés par décret en fonction de besoins politiques.

17 On trouve depuis plusieurs jours, sur les portes d'entrée et les murs de nombreuses facultés, la déclaration, code éthique, que le comité belgradois de l'association des professeurs d'université et scientifiques de Serbie a établi : nous ne voulons pas que d'autres administrent notre avenir ni qu'ils nous possèdent, c'est pourquoi nous n'acceptons pas de devenir les membres des comités administratifs et de surveillance, ni des recteurs et vices-recteurs nommés par ordonnance de la nouvelle loi. Nous donnons cette partie de l'autonomie personnelle en tant que défense contre les assauts contre l'autonomie de l'université.

Selon la nouvelle loi, le gouvernement de Serbie, sans aucune coopération des universités et des facultés, nomme les recteurs et les doyens, les membres des comités administratifs et de surveillance. Le pouvoir a le statut de propriétaire des universités et facultés, ainsi que de tous ceux qui travaillent à l'université. Les experts juridiques s'accordent : il n'existe nulle part dans le monde une telle ordonnance.

Un groupe de 16 professeurs de la Faculté de droit à Belgrade rend public qu'il répugne et refuse de signer la "déclaration de loyauté". Il a déjà envoyé à la cour constitutionnelle de Serbie un recours sur la légalité et la constitutionnalité de la loi sur l'université. Le syndicat de la Faculté d'électro-technique à Belgrade en a fait de même.

\section{Le litigieux article 165}

C'est l'article 165 de la loi sur l'université qui a suscité la plus grande agitation dans les cercles académiques. Il édicte que les salariés de l'université doivent conclure un contrat de travail dans les soixante jours suivants l'entrée en vigueur de la loi. La manière dont est fixée la date dans l'article 165 est anticonstitutionnelle et illégale, affirment les experts juridiques. Il y a encore des lacunes dans cette disposition : elle ne mentionne pas que l'employeur a l'obligation de proposer à tous les salariés de l'université un nouveau contrat de travail. Le recteur omnipotent, ayant obtenu une délégation de pouvoir du directeur d'entreprise, décide, arbitrairement, avec quels 
salariés il signe le contrat de travail, et avec lesquels il ne le fait pas. Cette disposition est illégale et en opposition directe avec la loi sur les relations de travail en Serbie. Les dispositions de l'article 165 de la loi sur l'université sont, par conséquent, anticonstitutionnelles, une seule partie pouvant se rétracter, commentent les spécialistes.

La loi sur l'université contient de nombreux vides légaux et juridiques, ce qui ouvre la porte à toutes sortes de manipulations et d'abus, et qui mène, inéluctablement, l'université à l'impasse la plus sombre.

La constitution de la Serbie garantit la liberté scientifique, que nie cette nouvelle loi sur l'université : « le recteur est l'organe gérant de l'université. Il a le droit et le devoir d'un directeur d'entreprise. L'administration de l'université à l'instar de celle d'une entreprise qui est une personne juridique pouvant réaliser des profits, tue la liberté de création, car, si la création scientifique doit être rentable, on ne peut plus parler de liberté ", déclarent les 16 professeurs de la Faculté de droit de Belgrade dans leur recours. L'établissement d'une similarité entre direction des universités et administration des entreprises aboutit à un choix totalement arbitraire des enseignants et collaborateurs scientifiques: le recteur ne doit-il pas choisir les candidats qui répondent aux conditions du concours, ne doit-il pas motiver sa décision, ne doit-il pas considérer l'avis de la commission électorale ? Le pouvoir discrétionnaire du recteur est réellement immense : il ouvre l'espace à l'élimination des insubordonnés et à la promotion de ceux qui répondent aux critères politico-moraux. Les initiateurs de la procédure devant la cour constitutionnelle de Serbie soulignent qu'ils vont recourir à l'Organisation Internationale du Travail, qui a déjà eu l'occasion de se prononcer sur l'adoption de critères politico-moraux comme condition des relations de travail.

\section{Loi imparfaite}

Les dispositions de l'article 165 représentent ce qu'on appelle une lex imperfecta, aucune sanction n'est prévue en cas de non signature. Cette norme est juridiquement inutile et absurde. Tous les salariés ont déjà institué des relations de travail valides. Pourtant, étant donné que cet État n'est certainement pas un État de droit, personne ne peut garantir que les nouveaux recteurs ne recevront pas des directives du Ministère de la culture pour interpréter autrement ces dispositions, c'est-à-dire qu'un refus de signer implique le licenciement. Dans ce cas, on peut toujours porter devant le tribunal le contentieux du travail, explique la professeur de la Faculté du droit à Belgrade, Vesna Rakić-Vodinelić.

Zoran Ivošević, président de l'association des juges de Serbie l'estime également : «si j'étais enseignant ou collaborateur à l'université, je ne signerais pas la déclaration de loyauté ». Si cette loi devait s'imposer à l'université, puiqu'on ne peut pas l'appliquer, elle anéantirait l'université. Il n'existe pas d'ordre juridique dans lequel cette loi pourrait être appliquée, observent de nombreux professeurs de l'université de Belgrade.

Les étincelles de résistance qui apparaissent dans de nombreuses facultés, de même qu'en dehors, réussiront-elles à empêcher l'application de la loi la plus restrictive de toute l'histoire de l'université ? L'université aura-t-elle la ténacité de la Faculté de 
philosophie de Belgrade, qui, à la fin des années 1970, s'était opposée à l'exclusion du groupe de philosophes, professeurs politiquement incorrects?

En ce qui concerne les enseignants et salariés des universités en Serbie, comment vontils réagir à l'humiliation, et aux dispositions catastrophiques, de par ses conséquences, de la nouvelle loi sur l'université ? À cette question essentielle, à ce dilemme moral individuel, chacun devra y répondre seul.

Les étudiants ont, lors des manifestations de 1996-1997, scandé: "Allons, tous à l'assaut !». Maintenant, les pancartes, qu'ils ont collées sur les murs de leurs facultés, invitent à se dresser face à la nouvelle loi sur l'université. Mais leur slogan est désormais: "Allons, tournons-nous vers l'ouest!». Manifestement, même cette génération a l'intention de rejoindre cette rivière sans retour qui a abandonné le pays lors de la "guerre à laquelle la Yougoslavie n'a pas pris part".

\section{AUTEURS}

\section{SLOBODANKA AST}

S. Ast est journaliste à Vreme. 